\title{
CLINICAL OUTCOMES OF ACCELERATED EPITHELIUM-OFF CORNEAL COLLAGEN CROSS-LINKING FOR: A CASE OF ULTRATHIN POSTLASIK ECTATIC CORNEA
}

\author{
Mounir, A. ${ }^{(*)}$, Korishy, M. \& Mostafa, E. \\ Ophthalmology dept., Faculty of Medicine, Sohag Univ., Sohag, Egypt \\ *E-mail:dramrmonir@yahoo.com
}

\begin{abstract}
29-year-old male with left eye diagnosed as post Lasik ectasia with extremely thin cornea after 3 years of uneventful Lasik surgery diagnosed by Scheimpflug based tomography device (Oculus Inc., Wetzlar, Germany), The uncorrected visual acuity (UCVA) in the left eye (LE) was 0.1 in decimal corrected to 0.4 by a refraction of -6.75Ds -4.5 0Dc @120, while the UCVA in the right eye (RE) was 1 in decimal without correction. Accelerated epithelium-off corneal cross-linking with pachymetricguided corneal epithelial removal by (Avedro, USA) was done for the affected eye. The patient was followed up for one year with improvement of visual acuity as regards UCVA and BCVA and improvement of corneal topographic parameters including keratometry including mean $K, K$ max and Belin $A B C D$ keratoconus staging.
\end{abstract}

Keywords: Epithelium-off corneal collagen cross-linking, Post Lasik ectasia, Ultrathin cornea

\section{Introduction}

Ectasias post corneal refractive surgery is caused by non-inflammatory biomechanical weakening of the cornea, with associated thinning and protrusion [1]. It is manifested by deterioration in visual acuity due to progressive myopia and irregular astigmatism caused by increasing corneal curvature [2,3]. Deep laser ablation with myopia $>8 \mathrm{D}$ and an increased Percentage Thickness Ablation (PTA) were the commonest risk factors for ectasia development in addition to preoperative corneal pachymetry $<500 \mu \mathrm{m}$ and eyes with preexisting topographic abnormalities like forme fruste keratoconus and pellucid marginal degeneration [4,5]. Treatment of ectasia includes various management modalities including glasses, hard contact lenses, Intracorneal ring segments (ICRS) and Corneal Cross-Linking (CXL) [6]. CXL has been approved and is being used in routine basis in for both keratoconus and ectasia after LASIK [7]. As one line of management, corneal collagen cross linking has been proved to be an efficient in halting the ectatic pathological process in the majority of patients with progressive keratoconus [8]. Standard CXL treatment 
with epithelial debridement is contraindicated in the presence of corneal thickness of less than 400 um as it may cause irreversible endothelial damage [9]. Accelerated corneal collagen cross linking was developed to maintain total irradiance with accelerated treatment time by total

\section{Case Presentation}

29-year-old male patient presented to the Cornea clinic of the Sohag Center for Lasik and Corneal Surgeries, Sohag, Egypt. The patient complained of left decreased vision after 3 years of Lasik eye surgery due to recent developing error of refraction. The uncorrected visual acuity (UCVA) in the left eye (LE) was 0.01 in decimal corrected to 0.4 by a refraction of -6.75Ds -4.5 0Dc @120, while the UCVA in the right eye (RE) was 1 in decimal without correction. Slit-lamp examination (RM-8900, TOPCON, Japan) of the anterior segment was normal in both eyes with bilateral clear cornea. There were no detected corneal scars or ghost vessels. In both eyes, there were normal corneal diameter and normal scleral color, with no edema or endothelial abnormalities. No iris abnormalities or lens opacities were detected. The fundus examination was performed, and it was normal. There was no positive family history of ectatic corneal disorders or systemic metabolic disorders like diabetes mellitus. Left corneal tomography was performed with corneal tomographic examination by Scheimpflug based tomography device (Oculus Inc., Wetzlar, Germany), which revealed a central cone with steep cornea (mean $\mathrm{K}$ : 45.6 um, Max K: 52.6 um). Central corneal thickness was extremely thin with thinnest location: 370 um and 375 um at the apex. Anterior and posterior elevations were high (+30 um, +70 um), fig. (1-a). Corneal tomography of the irradiation increase [10]. In this case report, we presented the clinical outcomes of accelerated epithelium-Off corneal collagen cross-linking after pachymetric-guided corneal epithelial removal for a case of ultrathin postlasik ectatic cornea.

right eye was normal while the left eye was diagnosed as post Lasik ectasia. The decision was to do accelerated epitheliumoff corneal collagen cross-linking for the left eye. Corneal epithelium was mechanically removed with pachymetric-guided corneal epithelial removal. The corneal surface was treated first by Vibex rapid (Isotonic $0.1 \%$ riboflavin with hydroxypropylmethylcellulose) for 15 mins which was applied every $90 \mathrm{~s}$ during the soak time. The treated corneal stroma and anterior chamber saturation were confirmed by slitlamp examination. Then the cornea was treated by the KXL® System accelerated CXL (Avedro) for 3 minutes using the pulsed mode with $30 \mathrm{~m} \mathrm{~W} / \mathrm{cm}^{2}$ power and total dose intensity: $5.4 \mathrm{~J} / \mathrm{cm}^{2}$. A soft contact lens bandage was applied at the end of surgery. The postoperative medication included topical antibiotics eye drops (e.g. moxifloxacin hydrochloride $0.5 \% 5$ times/ day for one week), topical steroid eye drops (e.g. Prednisolone acetate $1 \% 5$ times/day for one week, Lubricant eye drops and systemic non-steroidal anti-inflammatory drugs. The soft contact lens bandage was removed after 1 week. The patient was followed up for one year (Every 3 months). The follow up showed improvement in UCVA and BCVA together with corneal tomographic parameters improvement including Kmax, $\mathrm{K}$ mean and ABCD Belin, the results are summarized in tab. (1) and shows in figs. $(1-b, c, d)$. 
Table 1: The follow up of the refractive and topographic changes in Left eye after accelerated epi-off CXL through one year by Pentacam Scheimpflug device (Oculus GmBH, Wetzlar, Germany)

\begin{tabular}{|l|l|c|c|c|c|c|c|}
\hline \multicolumn{1}{|c|}{ Refraction } & $\begin{array}{c}\text { LCV } \\
\boldsymbol{A}\end{array}$ & $\begin{array}{c}\text { BCV } \\
\boldsymbol{A}\end{array}$ & Mean $K$ & K Max & $\begin{array}{c}\text { Thinnest } \\
\text { Location }\end{array}$ & $\begin{array}{c}\text { ABCD Belin } \\
\text { staging }\end{array}$ \\
\hline Preoperative & $\begin{array}{l}-6.75 \mathrm{Ds} \\
-4.50 \mathrm{Dc} @ 120 .\end{array}$ & 0.01 & 0.3 & $47.00 \mathrm{D}$ & $56.1 \mathrm{D}$ & $367 \mathrm{um}$ & $\mathrm{A} 3 \mathrm{~B} 5+\mathrm{C} 3 \mathrm{D} 4$ \\
$\mathbf{3}^{\text {rd }}$ month post-CXL & $\begin{array}{l}-4.00 \mathrm{Ds} \\
-3.50 \mathrm{Dc} @ 135 .\end{array}$ & 0.1 & 0.3 & $46.60 \mathrm{D}$ & $54.72 \mathrm{D}$ & $381 \mathrm{um}$ & $\mathrm{A} 2 \mathrm{~B} 4+\mathrm{C} 3 \mathrm{D} 3$ \\
$\mathbf{6}^{\text {th }}$ month post-CXL & $\begin{array}{l}-3.25 \mathrm{Ds} \\
-3.00 \mathrm{Dc} @ 133 .\end{array}$ & 0.1 & 0.4 & $45.60 \mathrm{D}$ & $52.44 \mathrm{D}$ & $370 \mathrm{um}$ & $\mathrm{A} 2 \mathrm{~B} 4+\mathrm{C} 3 \mathrm{D} 2$ \\
$\mathbf{1 2}^{\text {th }}$ month post-CXL & $\begin{array}{l}-2.75 \mathrm{Ds} \\
-1.75 \mathrm{Dc} @ 135 .\end{array}$ & 0.3 & 0.6 & $44.9 \mathrm{D}$ & $50.3 \mathrm{D}$ & $370 \mathrm{um}$ & $\mathrm{A} 1 \mathrm{~B} 4+\mathrm{C} 3 \mathrm{D} 2$ \\
\hline
\end{tabular}

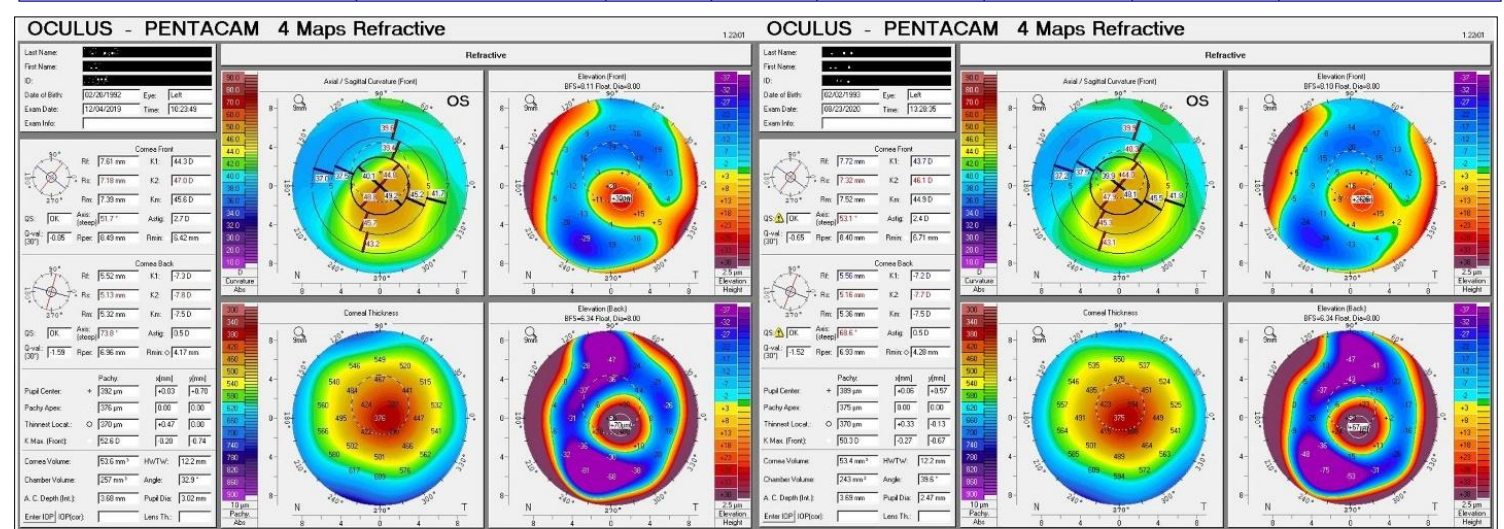

(A) Pre CXL

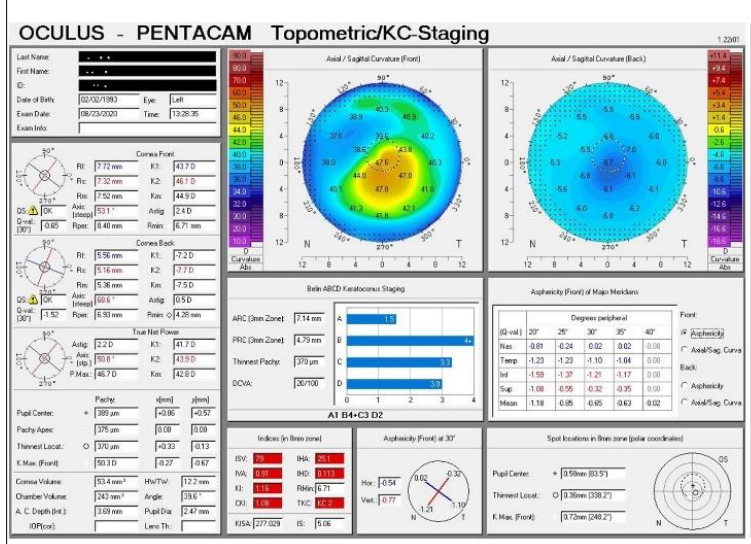

(C) Post one year CXL
(B) Post one year CXL

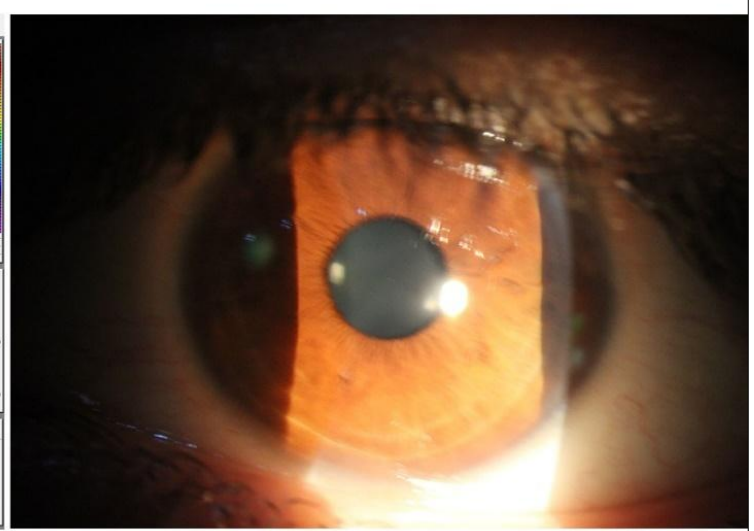

(D) Post one year CXL

Figure (1). a. Left 4 map printout of Pentacam Scheimpflug device (Oculus GmBH, Wetzlar, Germany) before accelerated epi-off CXL, $\underline{\mathbf{b}}$. Left 4 map printout of Pentacam Scheimpflug device (Oculus GmBH, Wetzlar, Germany) after one year of accelerated epi-off CXL, $\underline{\text {. }}$ Left Belin ABCD keratoconus staging after one year of accelerated epi-off CXL, $\underline{\mathbf{d}}$. Slitlamp photo of the left eye after one year of accelerated epi-off CXL

\section{Discussion}

Post-LASIK ectasia can be defined as a progressive corneal deformation causing a refractive and optical instability after uneventful laser in situ keratomileusis surgery [11]. The clinical signs of this complication include a combination of corneal stromal thinning, anterior and posterior corneal steepening, a progressive increase in myopia, irregular astigmatism, and corneal aberrations, followed by a progressive loss of corrected distance visual acuity [12-14]. In our case, post Lasik ectasia developed in one eye after 3 years of uneventful Lasik eye surgery presented 
with recent developing error of refraction. The treatment decision was to do corneal collagen crosslinking, but the main limitation of this treatment was the very low corneal thickness (367 um) after corneal tomographic examination. Usually, CXL is performed on eyes with a corneal thickness of at least $400 \mathrm{um}$ to avoid the cytotoxic impacts of the UVA on the corneal endothelium, lens, and other intraocular tissues [15]. So, standard CXL is considered a challenge in such ultrathin ectatic with previous Lasik surgery [16-17]. Multiple studies investigated CXL in such thin ectatic corneas, in a study of Kymionis GD et al, [18] they investigated a modified method for epithelial removal prior to CXL in 2 patients with regional thinning (less than 400 microns corresponding to the area of corneal steepening), they found no intra- or postoperative adverse events seen by the nine months follow up examination with stabilization of the corneal ectasia. Also, in a study of Hafezi F., et al, [19]. they investigated a modified treatment protocol by swelling thin corneas to a stromal thickness of at least $400 \mathrm{um}$ using hypoosmolar riboflavin solution. This treatment protocol was done in a case series of 20 patients, and no complications were observed. However, the use of this technique was limited due to increased intraoperative time and a relatively lower concentration of collagen in the hydrated stoma [20]. We avoided to use the transepithelial CXL treatment because of its low efficacy as compared to conventional treatment especially in stabilizing or improving keratometry [21]. After performing accelerated epithelium-off corneal collagen cross-linking, follow up was done for one year, no complications were detected and with improvement of visual and topographic parameters including ABCD Belin staging system which had been proven to be an effective method to reflect the anatomical changes seen in keratoconus and other ectatic diseases [22].

\section{Conclusion}

Accelerated epithelium-Off corneal collagen cross-linking after pachymetric-guided corneal epithelial removal was found to be safe and effective method in stabilizing and improving a case of ultrathin post Lasik ectatic cornea after one year follow up.

\section{References}

1. Chan C., Saad A., Randleman J., et al. Analysis of cases and accuracy of 3 risk scoring systems in predicting ectasia after laser in situ keratomileusis. J. Cataract Refract Surg. 2018; 44: 979-992.

2. Seiler T., Koufala K., Richter G. Iatrogenic keratectasia after laser in situ keratomileusis. J. Refract Surg. 1998; 14: 312-317.

3. Randleman J., Russell B. Ward M., et al. Risk factors and prognosis for corneal ectasia after LASIK. Ophthalmology. 2003; 110: 267-275.

4. Saad A., Binder P., Gatinel D. Evaluation of the percentage tissue altered as a risk factor for developing postlaser in situ keratomileusis ectasia. $\boldsymbol{J}$.
Cataract Refract Surg. 2017; 43: 946951.

5. O'Keefe M., Kirwan C. Laser epithelial keratomileusis in 2010-A review. Clin Exp Ophthalmol. 2010; 38:183-191.

6. Wolle M., Randleman J., Woodward M. Complications of refractive surgery: Ectasia after refractive surgery. Int Ophthalmol Clin. 2016; 56 (2): 127-139.

7. Yildirim A., Cakir H., Kara N., et al. Corneal collagen cross linking for ectasia after laser in situ keratomileusis: longterm results. J. of Cataract and Refractive Surgery. 2014; 40 (10): 1591-1596.

8. Hersh P., Stulting RD, Muller D., et al. United States multicenter clinical trial of corneal collagen cross linking for keratoconus treatment. Ophthalmology. 2017; 124: 1259-1270. 
9. Deshmukh R., Hafezi F., Kymionis G., et al. Current concepts in cross linking thin corneas. Indian J. Ophthalmol. 2019; 67 (1): 8-15.

10. Santhiago M. Accelerated corneal cross-linking: We must acquire knowledge as fast. J. Refract Surg. 2016; 32: 362-363.

11. Dupps Jr. Biomechanical modeling of corneal ectasia. J. Refract Surg 2005; 21: 186-190.

12. Pallikaris I., Kymionis G., Astyrakakis N. Corneal ectasia induced by laser in situ keratomileusis. J. Cataract Refract Surg. 2001; 27: 1796-1802.

13. Twa M., Nichols J., Joslin C., Characteristics of corneal ectasia after LASIK for myopia. Cornea 2004; 23: 447-457.

14. Ali JL., Shabayek M. Corneal higher order aberrations: A method to grade keratoconus. J. Refract Surg. 2006; 22: 539-545.

15. Cagil N., Sarac O., Can G., et al. Outcomes of corneal collagen cross linking using a customized epithelial debridement technique in keratoconic eyes with thin corneas. Int Ophthalmol. 2017; 37 (1): 103-109.

16. Wollensak G., Spoerl E., Rebel F., et al. Corneal endothelial cytotoxicity of
riboflavin/UVA treatment in vitro. Opthalmic Res. 2003; 35: 324-328.

17. Spoerl E., Wollensak G., Seiler T. Increased resistance of cross linked cornea agaist enzymatic difestion. Curr Eye Res. 2004; 29: 35-40.

18. Kymionis G., Diakonis V., Coskunseven E., Customized pachymetric guided epithelial debridement for corneal collagen cross linking. BMC Ophthalmol. 2009; 9: 10, doi: 10.1186/ 1471-2415-9-10.

19. Hafezi F., Mrochen M., Iseli H., et al. Collagen cross linking with ultravioletA and hypoosmolar riboflavin solution in thin corneas. J. Cataract Refract Surg. 2009; 35 (4): 621-624.

20. Sachdev G., Sachdev M. Recent advances in corneal collagen crosslinking. Indian J. Ophthalmol. 2017; 65 (9): 787-796.

21. Shalchi Z., Wang X., Nanavaty M. Safety and efficacy of epithelium removal and transepithelial corneal collagen cross linking for keratoconus. Eye (Lond) 2015; 29: 15-29.

22. Belin M., Kundu G., Shetty N., et al. ABCD: A new classification for keratoconus. Indian J. Ophthalmol. 2020; 68 (12): 2831-2834. 